\title{
Prévalence À L'obésité Par La Consommation Modérée D’huile De Palme Chez Le Rat
}

\author{
Alex Innocent Hollywale Bohué \\ Adama Kamagaté \\ Angoué Paul Yapo
}

Laboratoire de Physiologie animale, de Pharmacologie et de Phytothérapie,

UFR SN, Université Nangui Abrogoua, Abidjan, Côte d'Ivoire

doi: 10.19044/esj.2016.v12n30p224 URL:http://dx.doi.org/10.19044/esj.2016.v12n30p224

\begin{abstract}
Palm oil is a vegetable oil extracted from the pulp of the palm oil fruit. It comes in crude or refined form. It is an oil which caused controversy due to lack of enough information on its use. Our study has shown that moderate consumption of palm oil, that do not exceed $15 \%$ of food content, over 28 days does not cause a significant increase in body mass in young rats. Similarly, the lack of significance in the indices of food intake, digestibility, protein efficiency and energy balance, among control and experimental groups of the rats confirmed that palm oil is good for nutrition when its consumption is moderate. However, our study showed that moderate consumption of palm oil does not affect the total cholesterol, HDLcholesterol and LDL-cholesterol levels in young. While, moderate consumption of crude palm showed a significant increase in triglyceride rates but refined palm oil does not increase significantly triglyceride rates. Finally, our study showed that moderate consumption of palm oil does not cause a massive accumulation of the oil in the liver. This leads to say that palm oil is not the matter, but the way of consuming palm oil that could be a risk of metabolic syndromes.
\end{abstract}

Keywords: Palm oil, obesity, metabolic syndrome, triglycerides, cholesterol

\section{Résumé}

L'huile de palme est une huile végétale extraite de la pulpe des fruits du palmier à huile. Elle se présente sous forme brute ou raffinée. C’est une huile qui a entraîné des polémiques en raison du manque d'assez d'informations quant à son usage. Notre étude a montré qu'une consommation modérée de l'huile de palme, c'est à dire n'excèdent pas les 15\% de l'aliment, sur 28 jours n'entraîne pas une augmentation significative 
de la masse corporelle chez des rats jeunes. De même, l'absence de significativité au niveau des indices de consommation, de digestibilité, d'efficacité protéique et énergétique, entre les rats des lots témoin et expérimentaux confirme que l'huile de palme est bonne pour l'alimentation lorsque la consommation est modérée. Que ce soient les taux de cholestérol total, de HDL-cholestérol et de LDL-cholestérol notre étude a permis de montrer que chez le rat jeune une consommation modérée de l'huile de palme n’affecte pas les taux du cholestérol. Néanmoins nous avons constaté une augmentation significative du taux de triglycérides pour une consommation modérée d'huile de palme brute, par contre pour l'huile de palme raffinée ce taux de triglycérides n’augmente pas significativement pour une consommation modérée d'huile de palme raffinée. Enfin, notre étude montre que la consommation modérée de l’huile de palme n’entraîne pas une accumulation massive de cette huile au niveau du foie. Cela amène à dire que l'huile palme ne peut être mis en cause, mais c'est la façon de manger l'huile de palme qui peut être à l'origine des syndromes métaboliques.

Mots-clés : Huile de palme, obésité, syndrome métabolique, triglycérides, cholestérol

\section{Introduction}

Ces dernières décennies, l'industrialisation croissante et l'urbanisation galopante ont entraîné des bouleversements au niveau des régimes alimentaires, et des modes de vie des gens. Cela a eu un impact sur la santé et l'état nutritionnel des populations, en particulier celles dans les pays en développement (Organisation Mondiale de la Santé [OMS], 2003).

On assiste à la consommation d'aliments à forte densité énergétique, notamment ceux avec des teneurs élevées en acide gras saturés entraînant des depots sous forme de tissus adipeux (Shommo, et al, 2014), à l'origine des syndromes métaboliques. Les syndromes métaboliques regroupent un ensemble de maladies qui peuvent se retrouver chez un même individu, ce qui a donné naissance au concept de syndrome métabolique (SM) (Andersen, Wedderkopp, Hansen, Cooper, and Froberg, 2003 ; Milligan, Thompson, Vandongen, Beilin, and Burke, 1995 ; Raitakari, Porkka, Viikari, Ronnemaa, and Akerblom, 1994 ; Twisk, Kemper, Van Mechelen, and Post, 2001). Parmi celles-ci figure l'obésité abdominale considérée comme facteur de risque et facteur pathogénique du SM (Traxer, Safar, Daudon, and Haymann , 2006).

L’obésité qui était autrefois considérée comme une maladie propre aux sociétés occidentales et nombreux pays développés, augmente de façon 
spectaculaire dans les pays en développement, surtout en milieu urbain (OMS, 2003).

Aujourd'hui, l’obésité demeure un grave problème de santé publique à travers le monde (Correia, Pataky, and Golay, 2014), avec une prévalence qui a doublé entre 1980 et 2014. De nos jours, elle touche environ 13\% de la population adulte mondiale (OMS, 2015).

Suite à l'urbanisation croissante qui a entraîné une occidentalisation du mode de vie et certaines croyances socioculturelles qui encouragent la surcharge pondérale, l'obésité a atteint des proportions épidémiques et des niveaux supérieurs à 30\% chez les adultes, dans plusieurs pays d'Afrique (Correia, et al., 2014). En Côte d'Ivoire, l’obésité touche 7\% de la population adulte (Anonymous, 2014). De même, l’obésité et le surpoids touchent dans la société africaine de plus en plus de jeunes enfants (Mogre, Gaa, and Abukari, 2013)

Selon une étude actuelle, les pays les moins touchés par l’obésité sont la Corée et le Japon qui sont de grands consommateurs de poissons, qui sont très riche en acide gras polyinsaturés (AGPI) (Lee, Popkin, and Kim , 2002). Les lipides contenus dans le poisson et l'huile d'olive sont riches en AGPI, ce qui fait qu'ils sont moins stockés au niveau de l'organisme, donc responsable de ses effets contre l’obésité (Kim, Moon, and Popkin , 2002).

Cependant, l'on note une consommation plus accrue d'huile de palme, qui a une forte teneur en acides gras saturés (AGS), qui pourraient être responsables de la survenue de l’obésité (Chardigny, Malpuech-Brugere, and Sebedio 2005), en remplacement des huiles végétales hydrogénées et graisses animales. En effet, 80\% de la production mondiale d'huile de palme est utilisée dans l'alimentation (Omont, 2010).

C’est fort de ce constat que notre travail a été élaboré dans le but d'évaluer l'impact d'une consommation modérée d'huile de palme sur une période de 4 semaines chez le rat. Nous avons caractérisé de façon précise les troubles métaboliques en évaluant les paramètres lipidiques, la masse du foie, ainsi que les paramètres zootechniques, chez trois lots de rats soumis à différents régimes alimentaires.

\section{Matériel}

\section{Matériel animal et aliments expérimentaux}

Ces recherches ont été conduites sur 21 rats blancs albinos de l'espèce Rattus norvegicus de souche Wistar composés de mâles et de femelles, provenant de l'animalerie du Laboratoire de Physiologie, de Pharmacologie et de Pharmacopée de l’UFR-SN de l’Université Nangui Abrogoua. Ces rats avaient un âge compris entre 6 et 8 semaines, et une masse corporelle comprise entre 45 et $87 \mathrm{~g}$. Ces animaux sont nourris soit de 
granulés (FACI ${ }^{\circledR}$, Abidjan, Côte d'Ivoire) ou de granulés additionnés de lipides, et de l'eau de robinet à volonté.

Ces rats ont été répartis en 3 lots de 7 rats, ayant une masse moyenne homogène (des sous lots ont été constitués pour éviter des cas de gestation). Ils sont acclimatés dans les cages pendant 5 jours et sont soumis quotidiennement à la température de $25 \pm 2^{\circ} \mathrm{C}$, à 12 heures de lumière et 12 heures d'obscurité.

Pendant 4 semaines d'expérimentation, les rats sont nourris avec des granulés d'industrie, complémentés ou non avec de l'huile de palme brute ou raffinée, et de l'eau de robinet à volonté.

-Le lot 1 (lot témoin), d'une masse moyenne initiale de 64,50 \pm 5,84 g, reçoit une ration composée uniquement de granulés (G).

-Le lot 2 (lot traité), d'une masse moyenne initiale de 64,50 \pm 5,45 g, reçoit une ration composée de granulés, supplémentée à l'huile de palme brute $(\mathrm{G}+\mathrm{HB})$.

-Le lot 3 (lot traité), d'une masse moyenne initiale de 64,80 \pm 4,0 g, reçoit une ration composée de granulés, supplémentée à l'huile de palme raffinée $(\mathrm{G}+\mathrm{HR})$.

La composition du granulé est la suivante : 15\% de matière protéique brute, 3,5\% de matière grasse brute, $12 \%$ de matière cellulosique, $9 \%$ de matière minérale, $1 \%$ de calcium, $0,9 \%$ de phosphore, $0,3 \%$ de sodium, de vitamines, $\mathrm{A}, \mathrm{D}_{3}$, E et $\mathrm{B}$.

Toutes les expériences ont été menées selon un protocole qui respecte les conditions d'éthiques telles définies par les instances internationales tenant compte du bien-être des animaux de laboratoire, et ont été approuvées par un conseil scientifique de l’Université Nangui Abrogoua.

\section{Matériel technique}

\section{Réactifs}

Les réactifs utilisés lors de ces travaux sont composés entre autres d'alcool, d'éther.

\section{Matériel de laboratoire}

Ce matériel est composé de balance électronique, de centrifugeuse, de spectrophotomètre, de ciseaux, de tubes secs, de flacons, de coton, de cloche à anesthésie, et d’éprouvettes.

\section{Méthodes}

\section{Préparation des régimes alimentaires}

Des tests préliminaires ont été effectués en vue d’évaluer le pourcentage d'huile de palme contenu dans différents repas accompagnés de 
sauce graine de palme, achetés dans différents restaurants du district d’Abidjan, Côte d'Ivoire.

La proportion d'huile de palme estimée à 15\% par plat correspond à une consommation normale. Les granulés ont donc été supplémentés de cette proportion d'huile de palme pour les 2 lots expérimentaux.

\section{Prélèvements sanguins}

A la fin des quatre semaines de traitement, les animaux mis à jeûn la veille au soir, sont anesthésiés avec de l'éther et le sang des rats est collecté tôt le matin selon la technique d'amputation de $5 \mathrm{~mm}$ du bout de la queue après l'avoir désinfecté avec de l'alcool à $70^{\circ} \mathrm{C}$ (Kraus, 1980).

Le sang est recueilli dans des tubes secs qui sont mis dans une glacière contenant de la glace, puis acheminés au Centre de santé urbain, « La Grâce », situé dans la commune d’Abobo, Côte d’Ivoire. Le sang est centrifugé à 8000 tours/min pendant 3 min. Le sérum obtenu est conservé dans des tubes à $-20^{\circ} \mathrm{C}$ jusqu'au moment des analyses biochimiques.

\section{Analyses biochimiques}

Les analyses biochimiques sont faites à partir du sérum. Le cholestérol total (CT), le HDL-cholestérol (HDL-C), et les triglycérides (TG) sont déterminés par la méthode enzymatique et colorimétrique (Talke and Schubert, 1965). Ces paramètres sont déterminés en utilisant un spectrophotomètre de marque Robonik prietest.

Quant au LDL-cholestérol (LDL-C), il est calculé à partir de la formule de Friedwald ci-dessous:

$$
\text { LDL-C }=\text { CT }- \text { HDL-C }-\underline{\mathrm{TG}} \quad(\text { en g/l })
$$

\section{Contrôle de l'ingestion, de l'hydratation et de la croissance}

Le suivi de la croissance a été réalisé individuellement par une pesée journalière tous les jours entre 9h et 10h. Quant à l'ingestion et l'hydratation, elles ont été évaluées quotidiennement par groupe. Le suivi s’est fait par la détermination de la différence entre les quantités d'aliments et d'eau distribuées et les restants à l'aide d'une balance.

Ces pesées ont permis de déterminer le gain moyen de poids (GP), l'indice de consommation (IC) et l'indice d'hydratation (IH). De même l'efficacité protéique (EP) et l'efficacité énergétique (EE) ont été calculées selon les formules ci-dessous :

$\mathrm{GP}=$ masse de l'animal à une période - masse initiale 


$$
\begin{aligned}
& \text { IC = quantité d'aliments consommés durant une période } \\
& \text { gain de masse durant la même période }
\end{aligned}
$$

$$
\mathrm{IH}=\frac{\text { quantité d'eau consommée durant une période }}{\text { gain de masse durant la même période }}
$$

$\mathrm{EP}=$ quantité de protéines consommée durant une période gain de masse durant la même période

$\mathrm{EE}=$ quantité d'énergie consommée durant une période gain de masse durant la même période

\section{Mesure de la digestibilité}

La mesure de la digestibilité a été réalisée quotidiennement par groupe, par la collecte des crottes entre $9 \mathrm{~h}$ et $10 \mathrm{~h}$. L'excréta de chaque groupe a été séché sur un lit ventilé à $20^{\circ} \mathrm{C}$ pendant $24 \mathrm{~h}$, puis pesé. Ce qui a permis de déterminer l'indice de digestibilité (ID) selon la formule cidessous :

$$
\begin{aligned}
& \text { ID = (quantité d'aliments consommés - masse de crottes produites) durant une période } \\
& \text { gain de masse durant la même période. }
\end{aligned}
$$

\section{Mesure de la masse relative du foie}

Après 28 jours d'expérience les animaux ont été pesés avant d'être sacrifiés, et le foie de chaque rat a été prélevé soigneusement et pesé. Ce qui a permis de déterminer la masse relative du foie (MRF) selon la formule cidessous :

$$
\text { MRF }=\frac{\text { masse du foie de l'animal sacrifié }}{\text { masse de l'animal sacrifié }} 100
$$

\section{Analyses statistiques des résultats}

L'analyse statistique des données est faite grâce au logiciel GraphPad Prism 5.01 (San Diego, Californie, USA). Les résultats sont donnés sous forme de moyenne suivie de l'erreur standard sur la moyenne ( \pm SEM). Il est procédé au test de l'analyse de la variance à un facteur (ANOVA1) pour vérifier la normalité des variables. Lorsque des différences significatives sont 
révélées entre les moyennes testées, l’ANOVA1 est complétée par des comparaisons multiples des valeurs moyennes des différents paramètres en utilisant le test de Turkey-Kramer. Les différences sont considérées significatives à $\mathrm{P}<0,05$, hautement significatives à $\mathrm{P}<0,01$, et très hautement significatives à $\mathrm{P}<0,001$.

\section{Résultats}

\section{Effet des régimes alimentaires sur le gain de poids}

Pour étudier l'effet de l'huile de palme sur le gain de poids, nous avons utilisé 21 rats jeunes repartis en 3 lots de 7 rats. La Figure 1 indique les résultats relatifs au cumul du gain du poids corporel des rats témoins et expérimentaux par semaine, et sur 4 semaines. Au-delà de la $4{ }^{\text {ème }}$ semaine les cumuls de gain de poids pour les lots G, HB, HR sont, respectivement 57,5 \pm $10,1 \mathrm{~g}, 43,2 \pm 8,2 \mathrm{~g}$ et 34,8 $\pm 6,9 \mathrm{~g}$. L'analyse des données n'indique aucune différence significative entre les rats témoins (nourris uniquement aux granulés) et expérimentaux. Ce qui indique que la consommation modérée de l'huile de palme sur 28 jours n'a pas entraînée une augmentation significative de la masse corporelle des différents lots de rats. Pour mieux comprendre cela, nous avons analysé la consommation alimentaire des différents lots de rats pour voir si cela s'expliquait par un déséquilibre au niveau des quantités d'aliments consommées. La Figure 2 indique les résultats relatifs aux consommations alimentaires des rats témoins et expérimentaux par semaine, et sur 4 semaines. Les consommations alimentaires sur 28 jours pour les lots G, HB, HR sont, respectivement 12,6 $\pm 1,82$ g, $10,3 \pm 1,48$ et 10,3 $\pm 0,488$ g. L'analyse des données montre qu'au niveau de la consommation alimentaire, il n'y a pas de différence significative entre les rats du lot témoin et des lots expérimentaux. Donc nos données expliquent qu'autant il n'y a pas de différence significative au niveau du cumul de gain de poids pour les lots G, HB, HR, pour une consommation modérée d'huile de palme autant il n’y a pas également de différence significative au niveau des quantités d'aliments consommées sur la période de 28 jours chez des rats jeunes. 


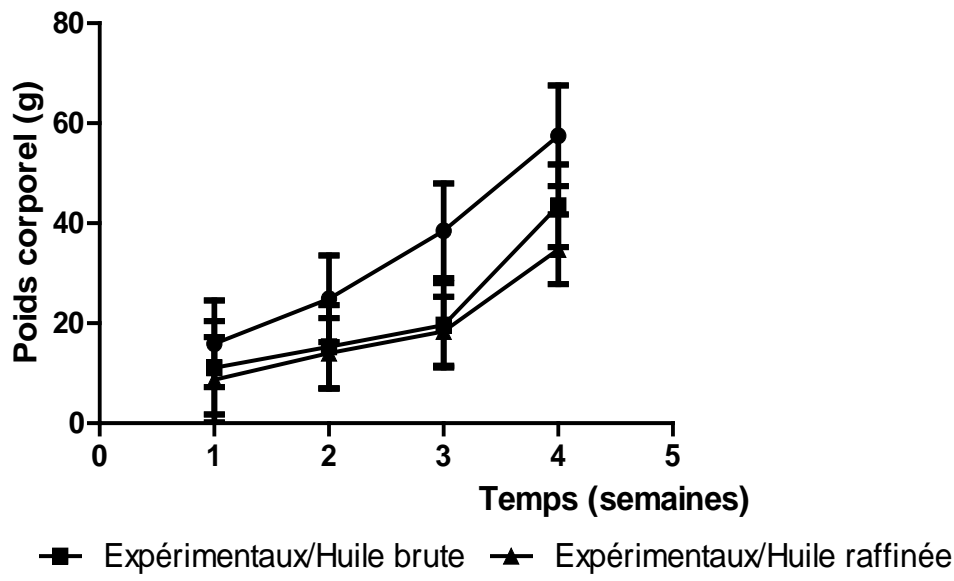

Figure 1: Evolution du poids corporel (g) chez les rats témoins et expérimentaux. Chaque valeur représente la moyenne \pm SEM, $n=7$ rats. La comparaison des moyennes est effectuée par le test de Turkey-Kramer.* $(\mathrm{p}<0,05)$ : Différence significative entre témoins et expérimentaux.

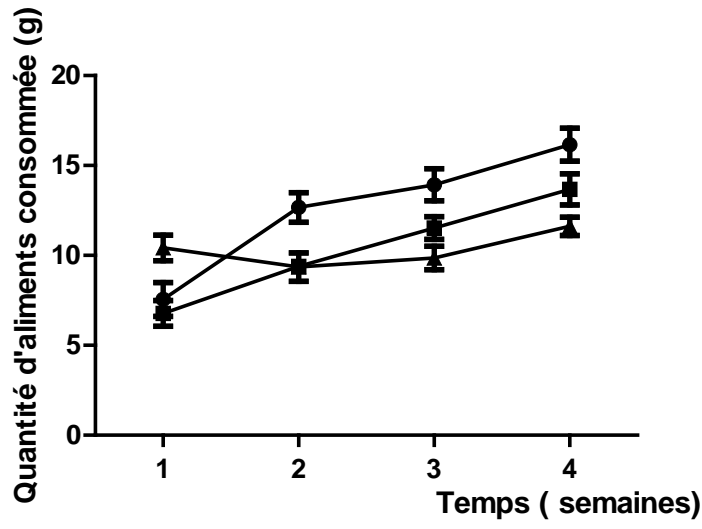

$\rightarrow$ Témoins

Expérimentaux/Huile brute

Expérimentaux/Huile raffinée

Figure 2 : Quantité d'aliment ingérée (g) des rats témoins et expérimentaux. Chaque valeur représente la moyenne \pm SEM, $n=7$ rats. La comparaison des moyennes est effectuée par le test de Turkey-Kramer. ${ }^{*}(\mathrm{p}<0,05)$ : Différence significative entre témoins et expérimentaux.

Effets des Régimes alimentaires sur les paramètres zootechniques : Le gain moyen de poids (GP), l'indice de consommation (IC), l'indice d'hydratation (IH), l'efficacité protéique (EP) et l'efficacité énergétique (EE))

Les effets de la consommation modérée de l'huile de palme sur une période de 28 jours chez les rats jeunes sur les paramètres zootechniques ont été évalués à travers le calcul de différents indices. Ces indices vont nous permettre de comparer les performances qu'on puisse réaliser avec les trois 
régimes alimentaires utilisés dans le cadre de cette étude sur les paramètres zootechniques.

L’indice de consommation nous permet d'accéder à l'impact qu'ont eu les trois régimes utilisés ici sur le gain de poids. La figure 3 renseigne sur les indices de consommation du lot témoin et des lots expérimentaux obtenus chez des rats jeunes sur une période de 28 jours. Nous avons obtenu pour valeurs après 28 jours de consommation d'aliments pour les lots G, HB, et $\mathrm{HR}$, respectivement 0,939 $\pm 0,194,1,58 \pm 0,583$ et 1,48 $\pm 0,333$. L’analyse des indices de consommations des lots $G, H B$ et $H R$ indique que la modification du régime alimentaire n’a pas eu un impact significatif sur le gain de poids.

Nous avons analysé également l'impact de la consommation d'eau sur le gain de poids. La figure 4 nous renseigne sur les indices d'hydratation du lot témoin et des lots expérimentaux obtenus chez des rats jeunes sur une période de 28 jours. Nous avons obtenu pour valeurs après 28 jours de consommation d'eau pour les lots $\mathrm{G}, \mathrm{HB}$, et $\mathrm{HR}$, respectivement 2,6 $\pm 0,464$, 3,8 \pm 1,38 et 3,6 $\pm 1,04$. L'analyse des indices d'hydratation des lots $\mathrm{G}, \mathrm{HB}$ et HR indique que la consommation d'eau n'a pas eu un impact significatif sur le gain de poids.

L’indice de digestibilité nous permet d'accéder à l’impact de la portion d'aliments absorbés par l'organisme des animaux sur le gain de poids. La figure 5 nous renseigne sur les indices de digestibilité du lot témoin et des lots expérimentaux obtenus chez des rats jeunes sur une période de 28 jours. Nous avons obtenu pour valeurs après 28 jours de consommation pour les lots $\mathrm{G}, \mathrm{HB}$, et $\mathrm{HR}$, respectivement 0,415 $\pm 0,112,0,815 \pm 0,305$ et 0,842 $\pm 0,161$. L'analyse des indices de digestibilités des lots $G, H B$ et HR indique que la digestibilité des aliments des différents régimes n’a pas eu un impact significatif sur le gain de poids.

L’indice d'efficacité protéique nous permet d'accéder à l'impact de la quantité de protéines contenues dans chaque régime alimentaire sur le gain de poids sur une période de 28 jours chez les rats jeunes. La figure 6 nous renseigne sur les efficacités protéiques des différents régimes sur le lot témoin et les lots expérimentaux obtenus chez des rats jeunes sur une période de 28 jours. Nous avons obtenu pour valeurs après 28 jours de consommation pour les lots $\mathrm{G}, \mathrm{HB}$, et $\mathrm{HR}$, respectivement 0,141 $\pm 0,029$, $0,201 \pm 0,074$ et $0,188 \pm 0,042$. L'analyse des indices d'efficacité protéique des lots $\mathrm{G}, \mathrm{HB}$ et $\mathrm{HR}$ indique que le taux de protéines contenu dans les différents régimes n’a pas eu un impact significatif sur le gain de poids.

Enfin l'indice d'efficacité énergétique nous permet d'accéder à l'impact du pouvoir calorique de chaque régime alimentaire sur le gain de poids sur une période de 28 jours chez les rats jeunes. La figure 7 nous renseigne sur l'indice d’efficacité énergétique des différents régimes sur le 
lot témoin et les lots expérimentaux obtenus chez des rats jeunes sur une période de 28 jours. Nous avons obtenu pour valeurs après 28 jours de consommation pour les lots $\mathrm{G}$, HB, et HR, respectivement 1,31 $\pm 0,271,4 \pm$ 1,48 et 3,75 $\pm 0,846$. L'analyse des indices d'efficacité énergétique des lots G, HB et HR indique que le pouvoir calorique des différents régimes n'a pas eu un impact significatif sur le gain de poids.

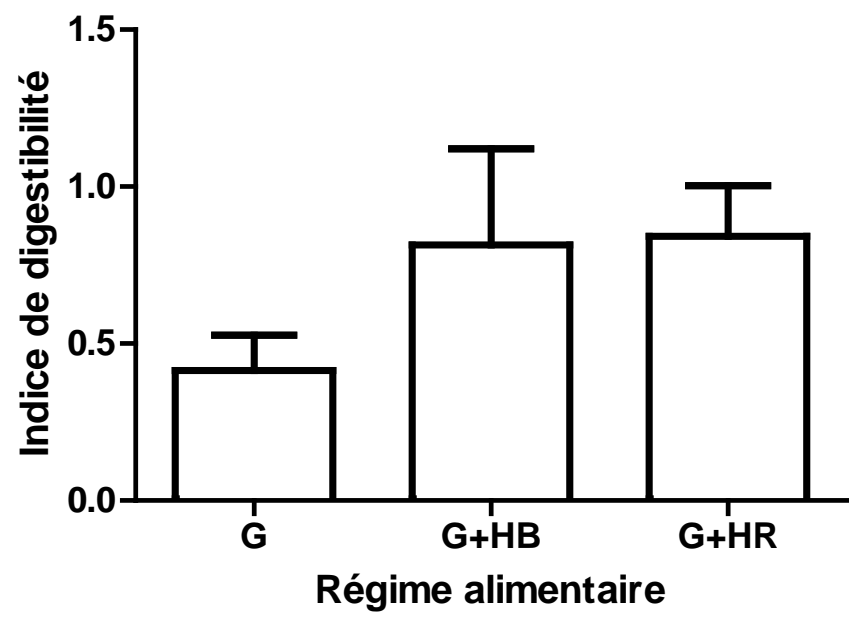

Figure 3 : Comparaison de l'Indice de consommation chez des rats soumis à différents régimes alimentaires. Chaque valeur représente la moyenne \pm SEM, $n=7$ rats. La comparaison des moyennes est effectuée par le test de Turkey-Kramer. $*(p<0,05)$ : Différence significative entre témoins et expérimentaux.

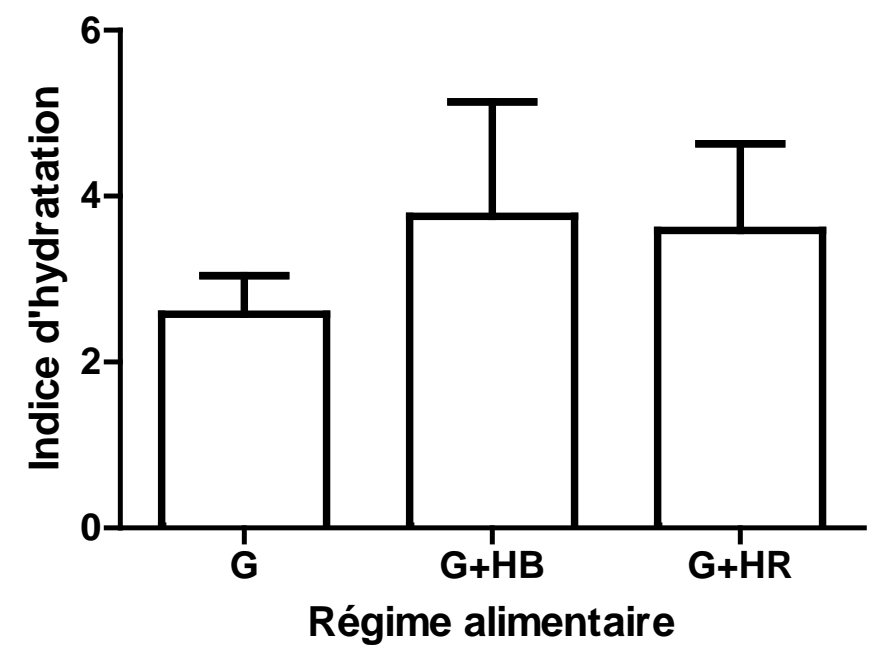

Figure 4 : Comparaison de l'Indice d'hydratation chez des rats soumis à différents régimes alimentaires. Chaque valeur représente la moyenne $\pm \mathrm{SEM}, \mathrm{n}=7$ rats. La comparaison des moyennes est effectuée par le test de Turkey-Kramer. ${ }^{*}(\mathrm{p}<0,05)$ : Différence significative entre témoins et expérimentaux. 


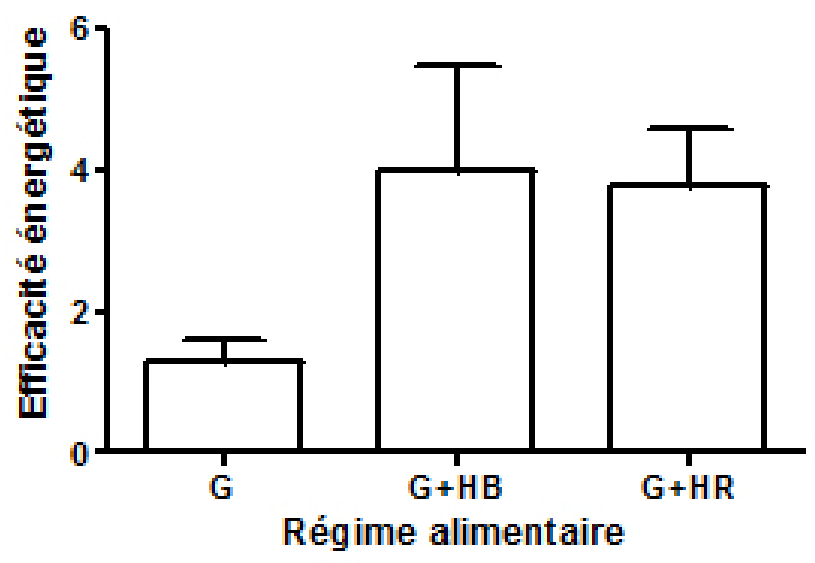

Figure 5 : Comparaison de l’Indice de digestibilité chez des rats soumis à différents régimes alimentaires. Chaque valeur représente la moyenne $\pm \mathrm{SEM}, \mathrm{n}=7$ rats. La comparaison des moyennes est effectuée par le test de Turkey-Kramer. * $(p<0,05)$ : Différence significative entre témoins et expérimentaux.

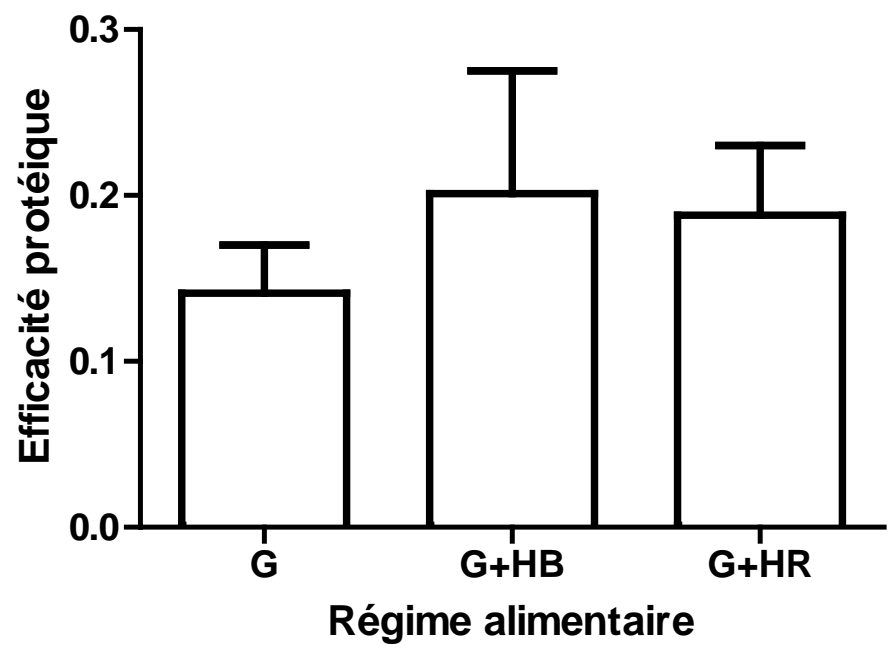

Figure 6 : Comparaison de l’Efficacité protéique chez des rats soumis à différents régimes alimentaires. Chaque valeur représente la moyenne $\pm S E M, n=7$ rats. La comparaison des moyennes est effectuée par le test de Turkey-Kramer. $*(p<0,05)$ : Différence significative entre témoins et expérimentaux. 


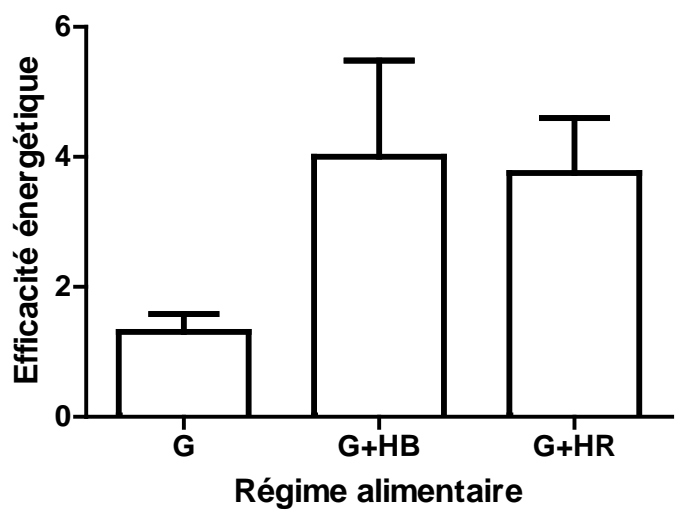

Figure 7 : Comparaison de l'Efficacité énergétique chez des rats soumis à différents régimes alimentaires. Chaque valeur représente la moyenne $\pm \mathrm{SEM}, \mathrm{n}=7$ rats. La comparaison des moyennes est effectuée par le test de Turkey-Kramer. ${ }^{*}(\mathrm{p}<0,05)$ : Différence significative entre témoins et expérimentaux.

\section{Effet des Régimes alimentaires sur les paramètres biochimiques}

Dans ce travail, il était important de connaître les taux du cholestérol total, du HDL-C, du LDL-C, et des triglycérides après 28 jours d'expérience chez les rats jeunes nourris avec des régimes alimentaires supplémentés avec de l'huile de palme. La figure 8 nous renseigne sur le cholestérol total du lot témoin et des lots expérimentaux en fonction des régimes alimentaires chez des rats jeunes après 28 jours. Nous avons obtenu pour valeurs pour les lots $\mathrm{G}, \mathrm{HB}$, et $\mathrm{HR}$, respectivement 46,98 $\pm 7,51 \mathrm{mg} / \mathrm{dl}, 39,82 \pm 4,93 \mathrm{mg} / \mathrm{dl}$ et 27,92 $\pm 4,55 \mathrm{mg} / \mathrm{dl}$. L'analyse des taux de cholestérol total des lots G, HB et HR indique que l'huile de palme n'a pas entraîné une augmentation significative $(p>0,05)$ du taux de cholestérol total.

Pour voir si la consommation d'huile de palme a un effet positif sur « le bon cholestérol » c'est à dire le HDL-C, nous avons dosé le taux de HDL-C chez les rats témoins et expérimentaux après 28 jours d'expérience. La figure 9 nous renseigne sur le taux de HDL-C du lot témoin et des lots expérimentaux en fonction des régimes alimentaires chez des rats jeunes après 28 jours d'expérience. Nous avons obtenu pour valeurs pour les lots $\mathrm{G}$, $\mathrm{HB}$, et HR, respectivement 60,9 \pm 6,78 mg/dl, $58 \pm 3,98 \mathrm{mg} / \mathrm{dl}$ et 52,2 \pm 7,56 $\mathrm{mg} / \mathrm{dl}$. L'analyse des taux de HDL-C des lots G, HB et HR indique que l'huile de palme n'a pas entraîné une augmentation significative du taux de HDL-C. Une augmentation du taux de HDL-C diminue le risque d'avoir le syndrome métabolique, ou a un effet bénéfique chez les patients avec le syndrome métabolique.

Ainsi nous avons essayé de voir si la consommation d'huile de palme a un effet positif sur « le mauvais cholestérol » c'est à dire le LDL-C. Nous avons dosé le taux de LDL-C chez les rats témoins et expérimentaux après 
28 jours d'expérience. La figure 10 nous renseigne sur le taux de LDL-C du lot témoin et des lots expérimentaux en fonction des régimes alimentaires chez des rats jeunes après 28 jours d'expérience. Nous avons obtenu pour valeurs pour les lots $\mathrm{G}, \mathrm{HB}$, et $\mathrm{HR}$, respectivement 39,3 $\pm 5,68 \mathrm{mg} / \mathrm{dl}, 56,4 \pm$ $6,01 \mathrm{mg} / \mathrm{dl}$ et $57,7 \pm 6,88 \mathrm{mg} / \mathrm{dl}$. L'analyse des taux de LDL-C des lots $\mathrm{G}$, HB et HR indique que l'huile de palme n'a pas entraîné une diminution significative $(p>0,05)$ du taux de LDL-C. Une diminution du taux de LDL-C diminue le risque d'avoir le syndrome métabolique, ou a un effet bénéfique chez les patients avec le syndrome métabolique.

Enfin, nous avons essayé de voir si la consommation d'huile de palme a un effet positif sur le taux de triglycérides. Nous avons donc dosé le taux sérique de triglycérides chez les rats témoins et expérimentaux après 28 jours d'expérience. La figure 11 nous renseigne sur le taux des triglycérides du lot témoin et des lots expérimentaux en fonction des régimes alimentaires chez des rats jeunes après 28 jours d'expérience. Nous avons obtenu pour valeurs pour les lots $\mathrm{G}, \mathrm{HB}$, et $\mathrm{HR}$, respectivement $127 \pm 22,4 \mathrm{mg} / \mathrm{dl}, 192 \pm$ 22,8 mg/dl et $167 \pm 18,9 \mathrm{mg} / \mathrm{dl}$. L'analyse du taux de triglycérides des rats du lot HB nourris aux granulés supplémentés d'huile de palme brute montre une augmentation significative du taux de triglycérides par rapport aux rats du lot $G(p<0,05)$ après 28 jours de consommation. Par contre, aucune différence significative $(p>0,05)$ de ce facteur n'est observée chez les rats du lot HR nourris aux granulés supplémentés avec de l'huile de palme raffinée par rapport aux rats du lot G. Les données indiquent que l'huile de palme brute a entraîné une augmentation significative du taux de triglycérides chez les rats jeunes après une consommation modérée de cette huile sur 28 jours. Un taux de triglycérides élevé pourrait indiquer la présence d'une dyslipidémie.

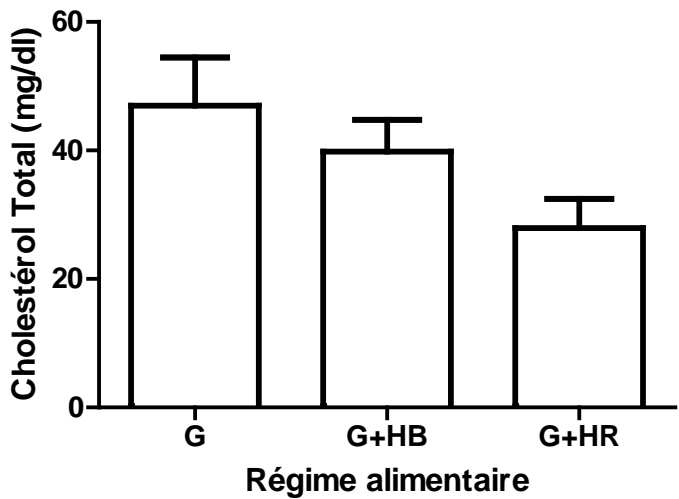

Figure 8 : Variation du taux de cholestérol total en fonction du régime alimentaire chez des rats. Chaque valeur représente la moyenne $\pm S E M, n=7$ rats. La comparaison des moyennes est effectuée par le test de Turkey-Kramer. ${ }^{*}(\mathrm{p}<0,05)$ : Différence significative entre témoins et expérimentaux. 


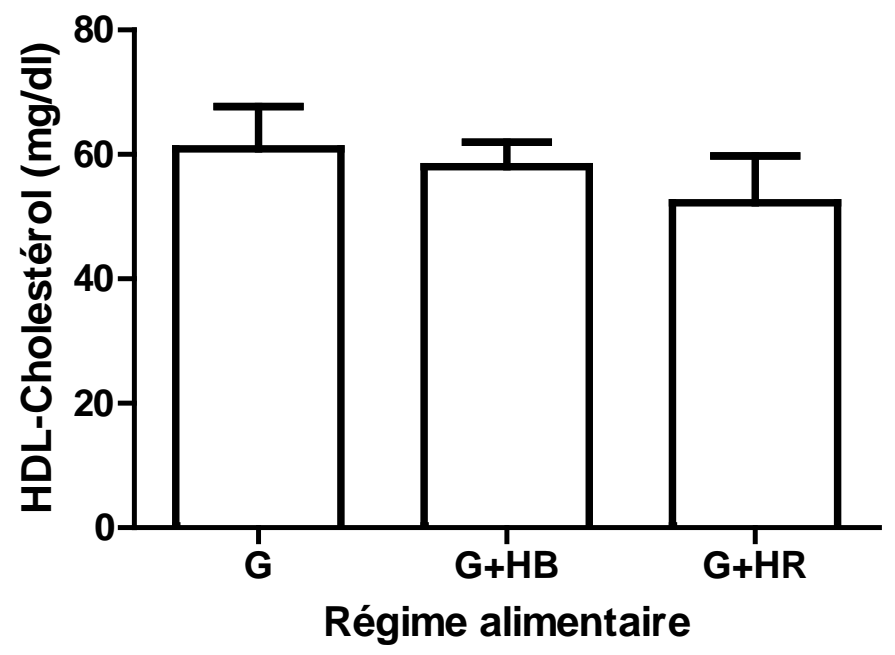

Figure 9 : Variation du taux de HDL-cholestérol en fonction du régime alimentaire chez des rats. Chaque valeur représente la moyenne $\pm E S, n=7$ rats. La comparaison des moyennes est effectuée par le test de Turkey-Kramer. ${ }^{*}(\mathrm{p}<0,05)$ : Différence significative entre témoins et expérimentaux.

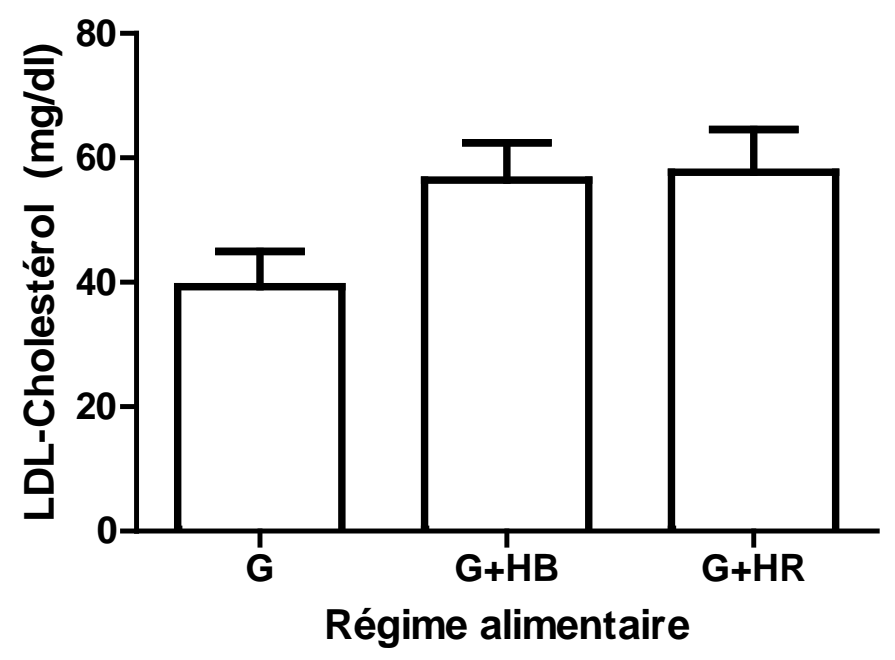

Figure 10 : Variation du taux de LDL-cholestérol en fonction du régime alimentaire chez des rats. Chaque valeur représente la moyenne \pm SEM, $n=7$ rats. La comparaison des moyennes est effectuée par le test de Turkey-Kramer. * $(\mathrm{p}<0,05)$ : Différence significative entre témoins et expérimentaux. 


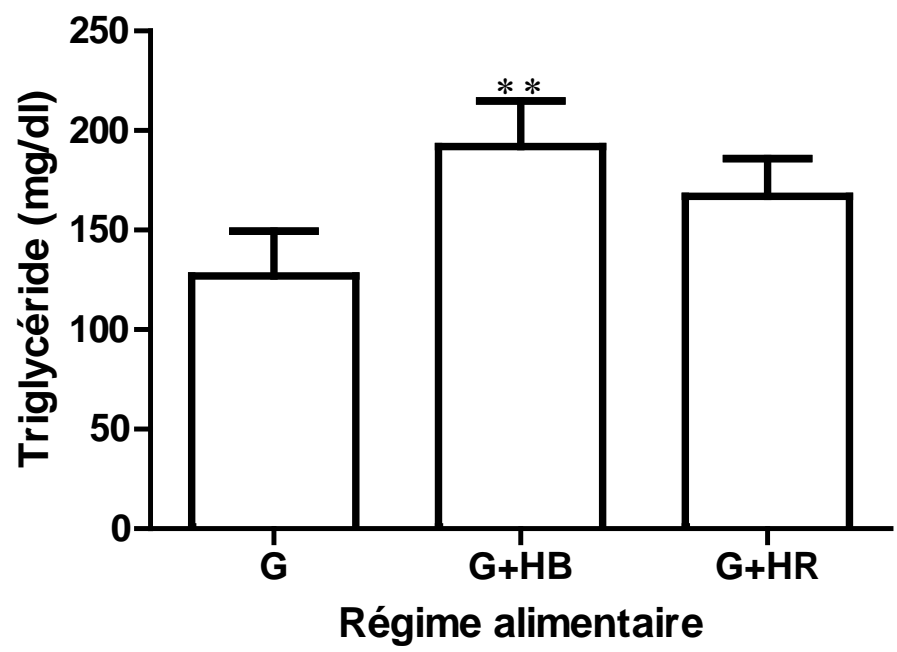

Figure 11 : Variation du taux de Triglycérides en fonction du régime alimentaire chez des rats. Chaque valeur représente la moyenne \pm SEM, n=7 rats. La comparaison des moyennes est effectuée par le test de Turkey-Kramer. ${ }^{* *}(\mathrm{p}<0,05)$ : Différence significative entre témoins et expérimentaux.

\section{Effet des régimes alimentaires sur le foie}

La figure 12 nous renseigne sur la masse relative du foie du lot témoin et des lots expérimentaux en fonction des régimes alimentaires chez des rats jeunes, après 28 jours d'expérience. Nous avons obtenu pour valeurs, en fin d'expérience pour les lots $\mathrm{G}, \mathrm{HB}$, et $\mathrm{HR}$, respectivement $0,036 \pm 0,001$ $0,038 \pm 0,002$ et $0,035 \pm 0,001$. L'analyse des masses relatives du foie des lots $\mathrm{G}, \mathrm{HB}$ et $\mathrm{HR}$ indique que la masse relative du foie ne varie pas significativement entre les rats témoins et les rats expérimentaux.

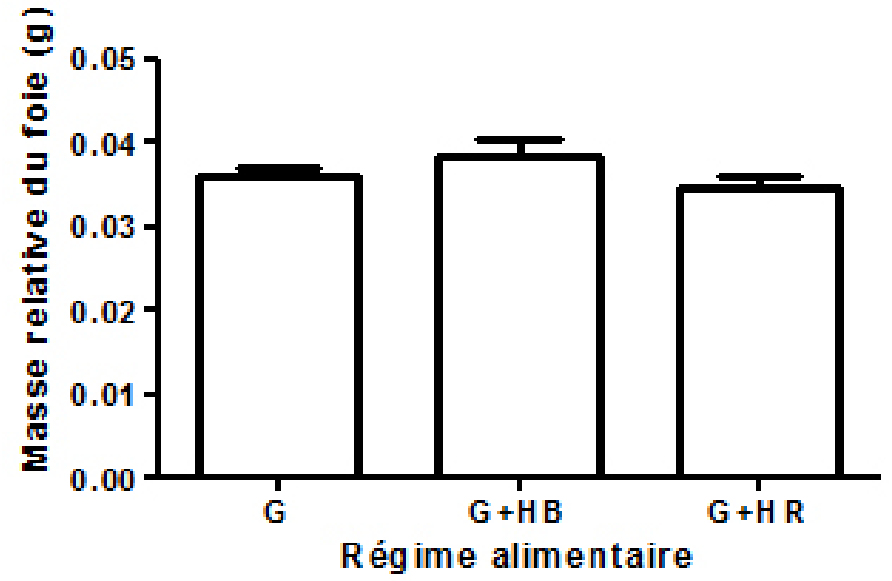

Figure 12 : Comparaison de la masse relative du foie chez des rats soumis à différents régimes alimentaires. Chaque valeur représente la moyenne \pm SEM, $n=7$ rats. La comparaison des moyennes est effectuée par le test de Turkey-Kramer. $*(p<0,05)$ : Différence significative entre témoins et expérimentaux. 


\section{Discussion}

Nous avons entrepris cette étude pour comprendre si dans des conditions normales de consommation de l'huile de palme on pouvait devenir obèse. L'huile de palme en Côte d'Ivoire rentre dans la composition de tous les mets traditionnels. C'est l'huile la plus consommée par la population. On en trouve en grande quantité dans des mets traditionnels comme le «garba», «l'alloco», la sauce graine. L'expérience a montré qu'une consommation modérée de l'huile de palme sur 28 jours n'a pas entraînée une augmentation significative de la masse corporelle chez des rats jeunes.

Ouattara, Amonkan, Meite, and Kati-Coulibaly (2014) ont fait une expérience similaire sur des rats adultes alors que nous avons utilisé des rats jeunes. Les poids initiaux de leurs rats sont environ deux fois supérieurs aux poids initiaux de nos rats. Leurs rats ont été gavés avec trois différentes huiles, à savoir l'huile de palme raffinée, l'huile d'olive et l'huile de Blighia sapida pendant 28 jours comme dans notre cas, avec des quantités d'environ $1 \mathrm{~g}, 2 \mathrm{~g}$ et $4 \mathrm{~g}$ par semaine.

Ces auteurs n'ont obtenu aucune différence significative entre les rats nourris aux huiles de palme, d'olive et de Blighia sapida pour une consommation d'environ $1 \mathrm{~g}$ par semaine sur 4 semaines. Par contre, ces auteurs ont obtenu une différence significative pour les rats qui ont été nourris à l'huile de palme raffinée, par rapport à l'huile d'olive et à l'huile de Blighia sapida pour une consommation d'environ $2 \mathrm{~g}$ et de $4 \mathrm{~g}$ par semaine sur 4 semaines.

Les résultats de cette étude confirment ceux obtenus dans ces précédents travaux quant aux consommations modérées d'huile de palme.

L'absence de significativité au niveau des indices de consommation, de digestibilité, d'efficacité protéique et énergétique entre les rats nourris aux granulés supplémentés d'huile de palme brute ou raffinée, et ceux nourris uniquement aux granulés, indique que l'huile de palme est bonne pour l'alimentation lorsque la consommation est modérée.

L'analyse des taux de cholestérol total, de HDL-cholestérol et LDLcholestérol des rats nourris aux granulés supplémentés à l'huile de palme brute ou raffinée, comparée aux taux observés chez les rats nourris uniquement aux granulés indique qu'une consommation modérée de l'huile de palme n'affecte pas le taux de cholestérol chez un individu. Cela amène à dire que l'huile palme ne peut être mise en cause, mais c'est la façon de la consommer qui peut être à l'origine des syndromes métaboliques.

Néanmoins nous avons constaté une augmentation significative du taux de triglycérides pour une consommation modérée d'huile de palme brute, par contre pour l'huile de palme raffinée ce taux de triglycérides n'augmente pas significativement pour une consommation modérée d'huile de palme raffinée. 
Messer (1995) affirme qu'une élévation significative du taux de triglycérides peut être le signe d'une hyperlipidémie.

Notre étude permet de dire que l'huile de palme raffinée pourrait être consommée modérément, par contre l'huile de palme brute communément appelée huile rouge par la population, doit être consommée beaucoup plus rarement.

Le foie joue un rôle majeur dans la régulation du métabolisme lipidique. Les résultats obtenus montrent qu'il n’existe pas de différence significative au niveau de la masse relative du foie chez les rats expérimentaux comparés à leurs témoins. De même, l’étude faite par Bravo, et al. (1998), suggère que la consommation de l'huile d'olive comparée à celle de l'huile de maïs n'entraîne aucune différence significative au niveau de la masse relative du foie chez les rats traités. Nos résultats indiquent que la consommation modérée de l'huile de palme n’entraîne pas une accumulation massive de cette huile au niveau du foie.

A terme, cette étude permettra de fixer des normes au niveau de la consommation d'huile de palme, c'est-à-dire que sa consommation doit se faire de façon modérée, c’est à dire n’excèdent pas les 15\% du poids total du repas, et qu'il faut donner la préférence à l'huile de palme raffinée par rapport à l'huile de palme brute.

\section{Conclusion}

Les acides gras libres sont utilisés comme carburant de second choix par l’organisme après le glucose. Les triglycérides sont formés de l'union d'un glycérol à trois acides gras et ils servent surtout de réserve d'énergie. Alors que les acides gras insaturés entre dans de nombreux processus biologique, les excédents d'acides gras saturés ont tendance à être stockés par l'organisme au niveau des tissus graisseux. Notre étude permet de dire qu'une consommation modérée d'huile de palme chez le rat n'induit pas significativement ni un gain de poids, ni une élévation du taux de cholestérol au niveau de l'organisme. Les indices de consommation, de digestibilité, d’efficacité protéique et énergétique vont dans le même sens pour l’huile de palme brute que pour l'huile de palme raffinée. Par contre, on a une élévation significative du taux de triglycérides avec l’huile de palme brute contrairement à l'huile de palme raffinée. Ainsi une accumulation massive de cette huile au niveau du foie n’a pas été constatée aussi bien pour une consommation modérée de l'huile de palme brute ou raffinée. Par conséquent, c'est la façon de consomme l'huile de palme qui peut être à l’origine des syndromes métaboliques. 


\section{References :}

1. Andersen, L. B., Wedderkopp, N., Hansen, H. S., Cooper, A. R., and Froberg, K. (2003). Biological cardiovascular risk factors cluster in danish children and adolescents: the european youth heart study. Prev Med, 37 , 363-367.

2. Anonymous (2014). Retrieved December 23, 2015 from

3. http://globalnutritionreport.org/files/2015/02/gnr14_cp_cote_divoire_ fr.pdf.

4. Bravo, E., Flora, L., Cantafora, A., De Luca, V., Tripodi, M., Avella, M., and Botham, K. M. (1998). The influence of dietary saturated and unsaturated fat on hepatic cholesterol metabolism and the biliary excretion of chylomicron cholesterol in the rat. Biochimica et Biophysica Acta, 1390 , 134-148.

5. Chardigny, J. M., Malpuech-Brugere, C., and Sebedio, J. L. (2005). Class, nature, origin and some metabolic aspects. Ocl, 12 , 111-115.

6. Correia, J., Pataky, Z., and Golay, A. (2014). Comprendre l’obésité en Afrique: poids du développement et des représentations. Rev Med Suisse, 423 , 712-716.

7. Kim, S. W., Moon S. J., and Popkin, B. M. (2002). The nutrition transition in South Korea. Am J Clin Nutr, 71 , 44-53.

8. Kraus, A. L. (1980). Research methodology, in H. J. Baker, J. R. Lindsey, and S. R. Weisbroth (eds), The Laboratory Rat (vol. 2 , pp. 1-30). New York : Academic Press.

9. Lee, M. J., Popkin, B. M., and Kim, S. (2002). The unique aspects of the nutrition transition in South Korea: the retention of healthful elements in their traditional diet. Public health Nutrition, 5 , 197-203.

10. Messer, N. T., (1995). Clinical pathology. Vet. Clin. Nor. Am. Eq. Pract., 11 (3) , 345-553.

11. Milligan R.A., Thompson C., Vandongen R., Beilin L.J., and Burke V. (1995). Clustering of cardiovascular risk factors in Australian adolescents: association with dietary excesses and deficiencies. $J$ Cardiovasc Risk, 2 , 515-523.

12. Mogre, V., Gaa, P. K., and R. N. S. (2013). Overweight, obesity and thinness and associated factors among school-aged children (5-14 years) in Tamale, northern Ghana. European Scientific Journal, 9 (20) , ISSN :1857- 7881 , e - ISSN 1857- 7431.

13. Omont, H. (2010). Contributions de la production d'huile de palme au développement Durable: problématique générale, controverses. Oléagineux Corps gras Lipides, 17 (6) , 362-367.

14. OMS (2015). Obésité et surpoids. Aide-mémoire N³11. Retrieved December 23, 2015 from http://www.who.int/mediacentre/factsheets/fs311/fr/ 
15. Organisation Mondiale de la Santé [OMS] (2003). Obésité: prévention et prise en charge de

16. l'épidémie mondiale. Rapport d'une consultation de l'OMS. Genève, Organisation mondiale de la Santé. (Série de rapports techniques, $\mathrm{N}^{\circ}$ 894). Retrieved December 23, 2015 from http://www.who.int/nutrition/publications/obesity/WHO_TRS_894/fr /

17. Ouattara, H., Amonkan, A. K., Meite, A., and Kati-Coulibaly, S. (2014). Comparaison de la biotolérance de l'huile extraite de l'arille de Blighiasapida (K. Koenig), des huiles de palme et d'olive chez le rat. Afrique Science, 10 (1) , 226-235.

18. Raitakari, O. T., Porkka, K. V., Viikari, J. S., Ronnemaa, T., and Akerblom, H. K. (1994). Clustering of risk factors for coronary heart disease in children and adolescents. The cardiovascular risk in young finns study. Acta Paediatr., 83 , 935-40.

19. Shommo, S. A. M., Aslami, I. M. A.,. Al-Tamimi, I. R. F., Anzi, G. F. K., Al Turki, R. S. And Al-Tamimi, K. M. (2014). The relationship between obesity and dietary intake of obese and preobese female students-Aja campus hail university. European Scientific Journal, 10 (24), ISSN :1857 - 7881, e - ISSN 18577431.

20. Talke, H. and Schubert, G. E. (1965). Enzymatic urea determination in the blood and serum in the Warburg optical test. Klin. Wochenschr., 43 , 174-175.

21. Traxer, O., Safar H., Daudon, M., and Haymann, J. P. (2006). Syndrome métabolique, obésité et lithiase urinaire. Progrès en Urologie, 16 , 418-420.

22. Twisk, J. W., Kemper, H. C., Van Mechelen, W., and Post, G. B. (2001). Clustering of risk factors for coronary heart disease. The longitudinal relationship with lifestyle. Ann. Epidemiol, 11, 157-65. 